Final published version: Timulak, L., \& Elliott, R. (2019). Taking Stock of DescriptiveInterpretative Qualitative Psychotherapy Research: Issues and Observations from the Front Line. Counselling \& Psychotherapy Research, 19, 8-15. DOI: 10.1002/capr.12197 (C)2019, Wiley. This is an author post-print and may not exactly replicate the final version. It is not the version of record.

\title{
Taking Stock of Descriptive-Interpretative Qualitative Psychotherapy Research: Issues and Observations from the Front Line
}

\author{
Ladislav Timulak ${ }^{1}$ and Robert Elliott ${ }^{2}$
}

1. Trinity College Dublin, Ireland,

2. University of Strathclyde, Glasgow, Scotland, United Kingdom

Author Note: Correspondence concerning this article should be addressed to Ladislav Timulak, School of Psychology, Trinity College Dublin, Dublin 2, Ireland, email: timulakl@,tcd.ie

\begin{abstract}
The paper offers a perspective on some of the methodological issues present in current qualitative psychotherapy research (QPR). The authors are involved in this type of research (for 20 and 40 years respectively) as researchers, as reviewers of papers submitted to journals publishing QPR, as well as past editors of such journals. The authors reflect at how the overall field of psychotherapy research has had an influence on how QPR is conducted. The authors further discuss a wide range of issues pertaining to QPR that often bring confusion in the community of psychotherapy researchers. These include: brand-naming largely overlapping qualitative methods; epistemological confusion arising from the context and application of these methods; issues of data collection and the range of types of qualitative data; the confounding of investigative questions or aims with findings; the issue of interpretative frameworks in data analysis; strategies by which findings are generated; difficulties in assessing the representativeness of findings to the target sample; issues of generalisability of findings; and possibilities for developing cumulative knowledge across studies. The paper specifically focuses on those genres of qualitative research that have a descriptive-interpretative character, typically represented by brand-name approaches such as empirical phenomenology, hermeneutics, interpretative phenomenological analysis, consensual qualitative research, grounded theory, thematic analysis, etc.
\end{abstract}


Taking Stock, p. 2

\section{Taking Stock of Descriptive-Interpretative Qualitative Psychotherapy Research: Issues and Observations from the Front Line}

At this point in the history of counselling and psychotherapy research, qualitative methods have become a well-established tradition. Qualitative psychotherapy research (QPR) first emerged in the 1980s, but only really established itself as a separate genre in the 1990s, when the work of researchers such as David Rennie, Robert Elliott, Bill Stiles, Clara Hill, and John McLeod found its way into mainstream scientific journals publishing psychotherapy research (including Journal of Counseling Psychology, Psychotherapy, Psychotherapy Research). These researchers, in line with developments in psychology and other disciplines such as education, nursing, sociology, explicitly referred to the methodological approaches they were using as qualitative. However, if we consider as qualitative research a flexible research approach that goes beyond mainstream hypothesis-testing (cf. Stiles, 2003), we can see that QPR has an even longer tradition than might be traditionally assumed. For example, early empirical studies on clients' experiences of therapy (Berzon, Pious, \& Farson, 1963; Bloch \& Reibstein, 1980; Bloch, Reibstein, Crouch, Holroyd, \& Themen, 1979; Lipkin, 1954; Orlinsky \& Howard; 1975; Strupp, Wallach, \& Wogan, 1964) often contained elements that would now be considered typical of qualitative methodology (for example an open-ended approach, flexibility, giving a voice to participants).

Since the 1980s, the number of qualitative research studies has grown significantly and QPR as a field has seen many new developments (Levitt, 2015); in particular, the field has seen the emergence of a number of different "brands" or varieties of QPR. Perhaps the earliest of these to appear have been Grounded Theory Analysis (GTA; Glaser \& Strauss, 1967), most closely associated with Rennie (Rennie, Phillips \& Quartaro, 1988), and Empirical Phenomenology (Wertz, 1983). These two original approaches then gave rise to several other branded approaches such as Hill, Thompson, and Williams' (1997) Consensual Qualitative Analysis (CQR). Similarities can be seen also in Smith, Flowers and Larkin's (2009) Interpretative Phenomenological Analysis (IPA), and the thematic analysis of Braun and Clarke (2006). In general, these approaches have labelled themselves as phenomenological, hermeneutic, interpretative and/or inductive and have emphasised client and therapist experience. In the psychotherapy field, other traditions of qualitative research, notably discourse-analysis (Potter, 1996), conversation analysis (ten Have, 1999) or critical-deconstructive approaches (Lather, 1991), have been largely neglected, although they have continued to develop in other areas of social and health science (for instance nursing). In this paper, while acknowledging the existence of these other streams of qualitative research, we will focus on this main stream of QPR, which we will refer to as descriptive-interpretative.

In this paper, we would like to comment on some of key issues that have emerged from the descriptive-interpretative tradition of QPR (Elliott \& Timulak, 2005). We would also like to offer our perspective on some of the methodological issues present in current QPR. Our perspective is based on our own experience as authors involved in this type of research (for 20 and 40 years respectively), our experience as reviewers of papers submitted to journals publishing QPR, as well as our experience as past editors of such journals. It is also worth noting we each operate out of broadly critical-realist or dialectical constructivist epistemologies and theories of method, largely because we find 
that these positions support our practice as counselling and psychotherapy researchers within a broadly humanistic-experiential theoretical orientation within psychotherapy.

We will primarily focus on qualitative research as conducted within the discipline of psychotherapy and we will look at how the overall field of psychotherapy research has had an influence on how QPR is conducted. We will then reflect on some aspects of conducting QPR where we believe there is confusion and/or a lack of clarity. These include a wide range of issues: brand-naming largely overlapping qualitative methods; epistemological confusion arising from the context and application of these methods; issues of data collection and the range of types of qualitative data; the confounding of investigative questions or aims with findings; the issue of interpretative frameworks in data analysis; strategies by which findings are generated; difficulties in assessing the representativeness of findings to the target sample; issues of generalisability of findings; and possibilities for developing cumulative knowledge across studies.

\section{Psychotherapy's Special Relationship to QPR}

Counselling/psychotherapy and qualitative research share a set of similarities that make them a good fit one another. Both take as their central method a procedure of specialised, structured but open-ended talk (McLeod, 2011) in which important experiences are symbolised; and both centrally involve empathy or deeply understanding the other (Rennie \& Fergus, 2006). Because of this natural (but imperfect) affinity, many psychotherapists are drawn more to qualitative research than to other kinds of research.

In turn, the nature of psychotherapy as a discipline shapes many aspects of QPR. To begin with, it shapes the type of research questions researchers ask (is therapy working; how is it working; does a particular theory of change work; what is the client's experience of particular aspects of therapeutic process such as transference, empathy). QPR is also shaped by the history of psychotherapy, psychotherapy research and related fields such as psychiatry and/or clinical and counselling psychology. There also exists a social organisation of science that is manifest in various forums and venues for communication of scientific findings. The tradition and the social organisation of psychotherapy means that it is a discipline that is dedicated to both scientific knowledge and professional application. This inevitably shapes what is studied, how it is studied, and how the research findings are communicated. The flagship journals in psychotherapy, psychiatry, and clinical/counselling psychology (Psychotherapy Research, Psychotherapy, Counselling and Psychotherapy Research, British Journal of Clinical Psychology, Psychology and Psychotherapy, Archives of General Psychiatry, Journal of Clinical and Consulting Psychology, Journal of Counseling Psychology), as well as the authors publishing in those journals (and beyond), shape standards regarding what is worth studying in psychotherapy, what methods are acceptable, and how studies should be presented. All of this has inevitably had an impact on the way QPR is conducted.

Receivers of QPR, such as practitioners, also shape that research. For instance, practitioners are interested to see if they can learn from research how to amend/improve their practice, and this influences how researchers conducting QPR communicate their findings. Fellow psychotherapy researchers represent a particular audience that may shape how QPR is conducted and presented, for example by asking questions such as whether the findings generated by a particular QPR study are credible, trustworthy, or valid. QPR researchers have wished to earn the trust of this larger community, which has 
informed choices about method, analysis or reporting. Broader stakeholders (including public health providers) outside the immediate therapist community have also shaped QPR, by directing influence or funding toward issues important to that community, including evidence-based provision and service delivery.

The influence of the applied discipline of psychotherapy on QPR sets it apart from qualitative research elsewhere in psychology. This is at times not fully reflected upon by psychotherapy researchers conducting qualitative studies when researchers eagerly adapt methods from other disciplines in a way that brings a risk of the adaptation not resonating with peers in the psychotherapy community. For instance, because of its traditional competition with biological interventions such as medication, randomised controlled trials and experimental studies are in general valued by the discipline of psychotherapy. This suggests that positivistic and post-positivistic perspectives (cf. Ponterotto, 2005) are in the mainstream of the discipline and thus influence the methodology and presentation of psychotherapy research, including qualitative research. This is something that a prospective qualitative researcher may want bear in mind when contemplating, conducting and presenting QPR.

\section{Brand-Name or Generic?}

Qualitative psychotherapy research, as with qualitative research in other disciplines, is often presented in the form of various brand-name approaches. For instance, in the tradition of descriptive-interpretative qualitative approaches (Elliott \& Timulak, 2005), we can find grounded theory, consensual qualitative research, empirical phenomenology, interpretative phenomenological analysis, thematic analysis. Some of these approaches were adapted from other disciplines (for instance, grounded theory from sociology), some were developed by psychotherapy researchers but are also used outside psychotherapy research (consensual qualitative research), some were developed from other areas of psychology but are sometimes used to study psychotherapy (interpretative phenomenological analysis), while others were developed by psychotherapy researchers and remain primarily employed in the field of psychotherapy research (for example comprehensive process analysis; Elliott, 1989).

As we have argued elsewhere (Elliott \& Timulak, 2005), these approaches share many aspects and can be seen, broadly speaking, as variations of the same method. While in some cases, these methods mainly focus on data analysis (thematic analysis), in general they share sets of procedures that shape sampling, data collection, data analysis/interpretation, and the presentation of results; and while they differ in some details (that can be thought of as brand specific), they essentially offer a variation of very similar procedures and guidelines.

The use of brand-named approaches is advantageous for researchers because reference to an 'established' method may allow the researcher gain credibility with editors, reviewers, and readers. By referencing an established method, the researcher can refer to an established set of procedures that are already described in other studies and in many cases also in methodological papers presenting the 'method' itself. Indeed, reviewers may expect that the method used by researchers in a study will be an established 'brand name' method. However, the branding of certain sets of procedures/guidelines, does not mean that those methods use some crucial features that will have distinct epistemological value and/or a distinct impact on findings when 
compared to other brands (Elliott \& Timulak, 2005). For instance, irrespective of brand name approach, an initial step in qualitative analysis commonly involves the dividing of text into separate meaning units, blocks of text, or lines, so that these can then later be named, thematised, clustered, categorised and so forth. This is common to descriptiveinterpretative approaches irrespective of brand name. Branding of the method may therefore be seen as more to do with issues of recognisability, tradition, or clarity, than with any decisive epistemological issues.

A related issue is that often there is a discrepancy between the reported use of a brand-named method (whether reported in order to gain credibility or for some other reason) and the actual used procedure, which may be more idiosyncratic or even inspired by a method of a different name. Indeed, a qualitative meta-method study (that is, a study examining methodological features) of qualitative studies of clients' experiences of therapy (Levitt, Pomerville, Surace, \& Grabowski, 2017) showed that studies reporting use of the same (brand) named method were not necessarily following the same procedures, while studies reporting use of different brand-named methods sometimes actually did share the same procedures. In other words, some epistemological and methodological features were shared or not shared regardless of the name of the method used.

We are pointing to this fact because in our experience the review of qualitative psychotherapy studies can at times focus on inconsequential aspects of methodological branding to the exclusion of researcher flexibility and creativity; a flexibility and creativity that might be influenced by and relevant to the subject of study. For instance, it has happened to us as authors that we were required by reviewers to identify which named procedure we had applied in a study in order that the procedure could be deemed credible. In such cases, we simply used a name that we were familiar with or felt we could justify as being broadly consistent with what we had done (we usually referred to our own method). At other times, reviewers criticised us for not using the method in the way that they thought it should be used, even though the method used was one developed and defined by ourselves.

We would argue therefore that descriptive-interpretative approaches simply describe and interpret certain phenomenon. The means of description and interpretation (Thorne, 2008) have to be transparently shared, and they have to be utilised systematically, optimally with a rigor (for example, checks) that enhances credibility and trustworthiness (Morrow, 2005). Many good examples of such procedures exist. Again, they are usually referred to by brand-names (interpretive phenomenological analysis, consensual qualitative research, comprehensive process analysis, empirical phenomenology, or grounded theory method); but, we argue, these methods are more similar than dissimilar, and insisting on a particular set of procedures or that procedures be limited to those defined by particular brand-names, is counterproductive for QPR. Further, we argue that referring solely to a brand name is insufficient as a description of one's qualitative approach; what is required is a more specific description of the actual research procedures followed.

\section{A Common Pitfall: Confusing Domains of Investigation with Findings}

We employ the word "domain" to refer to a broad organising category used by a qualitative researcher to organise their findings (Elliott \& Timulak, 2005; cf., Hill, 
Thompson \& Williams, 1997). Glaser (1978) referred to these as formal categories as opposed to substantive categories. In our view, the same idea is captured by the headings used in Strauss and Corbin's (1998) axial coding method. The problem here is that of tautologically confusing informant topics stemming from the researcher's aims and interview questions with findings. For instance, one studies helpful aspects of therapy (domain of investigation) and discovers 'helpful aspects of therapy' (finding).

This difficulty is not specific to QPR; in fact, it could be argued that it is simply an unconscious carry-over of hypothesis testing method of traditional positivist quantitative research. Nevertheless, it is perhaps more prevalent among studies conducted by students with limited experience conducting qualitative research and facing limited word counts. The examination of dissertations (and to a somewhat lesser extent papers submitted to research journals) all too often reveals that authors report finding what they set out to study. For example, a study wants to find out what constitutes a good supervision hour (domain of investigation) and the main finding captured in the name of the main category is 'a good supervision hour'. In such scenarios, authors do not discriminate between their domain(s) of investigation (given by their research or interview questions) and the actual findings that have arisen from that investigation. A variation of the difficulty occurs when a particular approach (for example, grounded theory) suggests that the study should result in a single core category or finding, pushing the researcher toward an overly broad or abstract label that is too general to be informative or useful. In other cases, this core finding is erroneously given a name that is indistinguishable from the stated research question (see the example of 'a good supervision hour' above) and the limited word count forces the researcher to edit out substantive informative details of their more specific findings.

In general, we argue that domains of investigation should be led by the research question(s), the data collection strategy (such as an interview schedule), and the constituents of the phenomenon under investigation that are relevant to the investigation. In psychotherapy research this might be differing perspectives (for instance, client, therapist, and observer perspectives); chronology (in studying significant therapy events one might look at their context, the processes within the event itself, and its later impacts), and/or theoretical framework utilised (assimilation of problematic experiences theory; Stiles, 2011). Several qualitative psychotherapy researchers clearly point to those procedures that distinguish between the broad domains of investigations and actual findings (often referred to as categories, themes and so forth). Thus, we can have a domain such as helpful impact of therapy and the actual findings (categories/themes) populating that domain, for instance, insight, feeling understood, feeling empowered. This distinction is crucial and offers not only conceptual clarity for the reader, but also builds the credibility of the study as readers can see that the authors are methodical and conceptually crisp and coherent.

The Role of Interpretative Frameworks in QPR: Error or Necessity? Again, although the relationship between description and interpretation in QPR analysis is not only an issue within psychotherapy research, it has particular importance in this context. Descriptive and interpretative approaches to qualitative research endeavour to keep a balance between the description of phenomena and the interpretation of the described phenomena. Given that researchers want to report concisely on what was found 
and given that we are by nature meaning-making individuals, it is inevitable that any findings/results will be coloured by an interpretative framework. However, it has rarely been clearly described where interpretations come from in QPR studies. For a notable exception, see Rennie (2000) who attempted to capture how categories come from the data ("induction"), often require an empathic or intuitive leap by the researcher ("abduction"), must ultimately resonate with the researcher's and audience's own experience and be assessed by them in their careful reading of their data or the examples given ("deduction").

Indeed, following examples from other disciplines that have utilised qualitative methodologies and perhaps in defiance of a mainstream positivistic perspective (with the inherent problems of the hypothetic-deductive system, including, observers' assumptions and values - see description in Stiles, 2003), QPR started to use 'bracketing' as a method by which researchers might prevent prior beliefs or values from influencing the phenomena under investigation (see for instance, Consensual Qualitative Research method [CQR; Hill, Thomson, \& Williams, 2007]). The findings (categories/themes) thus supposedly came from the data (or participants). As we have pointed out elsewhere (Elliott \& Timulak, 2005), we are sceptical about the usefulness of such an effort (see also an elaborate criticism by Stiles [2003]), as what an author refers to as bracketing may actually mean that an implicit, undeclared interpretative framework is operating. For example, one can easily imagine studies of client change processes in therapy conducted by humanistic or CBT qualitative researchers, all of whom 'bracket' their assumptions, but where the humanistic researcher sees clients as experiencing empowerment, while the CBT researcher sees the same clients as capable of challenging negative thinking. Indeed, examples from QPR show that the theoretical and personal background of authors shapes results, a phenomenon referred to by Strauss and Corbin (1998) as theoretical sensitivity. (For an example of CQR in which two separate teams arrived at different conclusions analysing the same data despite an effort to bracket their biases, see Ladany, Thompson, \& Hill, 2012.)

Although many researchers involved in QPR claim that they are giving a voice to participants (often clients), we argue that this is inevitably coloured by the researchers/ analysts' perspective (that is, their interpretative framework). Thus, we urge researchers to go beyond empty claims of bracketing in two ways: First, it is essential for them to clearly and transparently disclose what they have brought to the research by way of expectations or commitments (Elliott, Rennie \& Fischer, 1999; Levitt, Bamberg, Creswell, Frost, Josselson, \& Suárez-Orozco, 2018; Levitt, Motulsky, Wertz, Morrow, \& Ponterotto, 2017). Second, together with Stiles (2003), we stress the importance of permeability on the researchers' side so that researchers are open to changing their expectations and understandings the process of interacting with their data.

We also encourage qualitative researchers not to shy away from theoreticallyinformed qualitative studies, as exemplified by the work of Stiles (2011) on assimilation, or recent studies by Timulak's group (O'Brien et al., 2017). This approach starts from a clearly articulated and openly declared theoretical position. The point of such theoretically-driven psychotherapy research is not to test the theory but rather to elaborate and specify how the theory plays out in particular situations. Moreover, starting from such a framework allows researchers to read their data more deeply by setting up a kind of dialogue between their theoretically-driven psychotherapy interests and the 
informants' reported experiences. It provides a framework to organise the data while at the same time using the data to modify, adapt or in some cases even to break the organising theory. This is also the rational-empirical approach favoured by the qualitative exploratory phase of task analysis within a broader dialectic constructivist epistemology (Greenberg, 2007).

In our experience, this theory building (Stiles, 2011) approach to qualitative research can be met with misunderstanding or incomprehension, when reviewers confuse qualitative research with being atheoretical and require a bracketing or tabula rasa strategy as the only valid approach to qualitative research. By contrast, we argue that in any QPR analysis, some kind of interpretative framework is inevitably at play. We suggest that QPR researchers should make their framework transparent by spending time articulating it clearly both for themselves and for readers.

\section{Coping with Common Misunderstandings about QPR}

In our experience as authors undergoing review, reviewers and editors, we have also noticed a range of common recurrent misunderstandings of QPR than go beyond the broad issues just noted.

Are Therapy Sessions Qualitative Data? First, we have had the experience that a study examining therapy session recordings/transcripts was criticised for not being qualitative, because it did not use interviews as the form of data collection. Historically, qualitative research has always included data from observation. Indeed, the first grounded theory studies were primarily observational (Glaser \& Strauss, 1967). Observation-based QPR in particular draw on the long tradition of examining recordings of therapy (cf. Rogers, 1942). The in-depth analysis of therapy sessions is also very close to the discipline of psychotherapy. For instance, it is an important part of reflective practice and clinical supervision.

\section{Isn't Writing about "Results" or "Findings" a Symptom of a Positivist} Epistemology? In their qualitative publishability criteria article, Elliott et al. (1999) were careful to refer to the "Returns" of qualitative studies rather to "Results" or "Findings"; they did so in the face of criticisms from constructivist qualitative researchers that the latter terms reflect researcher assumptions that portray qualitative research either as an intervention that the researcher does to passive participants (thus resulting in "results"), or as an unearthing ("finding") pre-existing facts. However, over the past 20 years alternatives such as "returns" have never really caught on, and a recent informal survey we made indicates that the terms "Results" and "Findings" are both now firmly entrenched in qualitative research reports. However, the expanded usage of these terms to the products of qualitative research now means that their meaning has shifted so that in our view their use currently implies "what emerges from the dynamic interaction between the researcher/research method and the participants/participant experiences". The embedded epistemology is therefore, as Rennie (2000) argued, neither realist/positivist nor relativist/constructivist, but rather something in between (Rennie's "rocky middle road"), which can be characterised variously as critical-realist (Cook \& Campbell, 1979) or dialectically constructivist (Elliott et al., 2009).

Is it Acceptable and Useful to Enumerate Category Occurrences in Your Qualitative Data? We also notice a reluctance by some authors to report the extent to which certain categories are representative of the sample (that is, how many participants 
from the sample a finding pertains to, or how many meaning units exemplify a category). A criticism we encountered was that reporting this information meant embracing a positivistic paradigm. Yet, in our experience, those same studies that omit this information often refer to undisclosed numbers of participants as 'some', or 'few' or 'several', etc, as if quantifying with words was somehow different from quantifying with numbers. Given the context of contemporary psychotherapy and counselling, in which positivism has been largely supplanted by a range of post-positivist epistemologies from critical realism to various forms of constructivism and hermeneutic views (Barker, Pistrang \& Elliott, 2015), we do not see any harm in reporting information that the researchers already have (such as to how many participants a finding/theme/ category pertains to). It does not harm to share with the reader how well a finding is represented among participants, so that the reader can see that it is not only a particular participant (or a very small group of participants) that shapes what is being found. To share the representativeness of the findings to the sample with the reader contributes to credibility and trustworthiness as well as transparency of the analysis. More importantly, it is also useful for interpreting the significance or meaning of the categories found. For example, based on their frequency some categories are possibly essential constituents of the phenomenon being studied (occurring in all or almost all informants), while others may define a typical instance of it (occurring in at least half); still other categories may define meaningful variations (replicated in multiple informants but less than half); finally, some categories could define unique or idiosyncratic instances, at least within a given data set (Elliott et al., 1994; see also Hill et al., 1997). However, the point is not the sheer quantity of meaning units or informants that exemplify the finding, but rather the possible meaning of the finding, in terms of its representativeness within the sample.

Can we Generalise the Findings of Qualitative Studies? Related to the issue of representativeness is the issue of generalisability. Indeed, some authors shy away altogether from using any numbers in order not to confuse the reader with any suggestion of generalisability of their findings. It is common to come across QPR studies claiming that their findings are not 'generalizable'. This is obviously a problem given that in any research we want to be able to infer and apply findings to other people, contexts, situations, etc. That is why also in qualitative research we refer to 'sample' and not 'population' (or 'all people fulfilling a particular characteristic'). Although the logic of generalisability is different in qualitative research than in quantitative research, we still want to say something about what we are studying that goes beyond our particular sample. All social science research is interested in generalisable knowledge; that is after all, part of the definition of science (Cook \& Campbell, 1979). There is a question, however, of what kind of qualitative knowledge can be generalised. At a minimum, we can say that even a small qualitative study can show us what is possible. Making inferences about how likely something is a more difficult proposition, given the relatively small samples in qualitative research and given the logic of qualitative research that does not use statistical probabilities and confidence intervals to provide a basis for making inferences about populations. In qualitative research this inference is done 'qualitatively', which is why we need the sample to be well-situated (described). Bill Stiles (2003) refers to generalisability in the context of a qualitative study as transferability capturing the interpretative leap of transferring the findings from a study to other people, contexts, 
situations, or settings. (For an interesting discussion of generalisability in qualitative research in other discipline see for instance Smith, 2018).

How is QPR Handling Innovative Research? Because QPR is still relatively new and developing as a genre, new topics and methods are still emerging at a fairly quick pace, leading to complications in the publication process. The manner in which a study is presented is often shaped by and justified by reference to previous research (qualitative and quantitative) already published in the particular journals being considered by authors for their submission. This in turn is shaped by the discipline of psychotherapy and the culture of psychotherapy research publishing standards as we have described above. In some instances, however, authors of innovative papers may be at a disadvantage as they may be breaking new ground in an area where there is little or no previous research to draw upon, including prototypical studies. In such cases, authors should be prepared to encounter difficulties in having a paper accepted (it has happened to us on many occasions). Authors may see how reviewers or editors (in unsuccessful submissions or earlier rounds of review) read the paper and in future submissions preemptively mitigate against potential misunderstandings of the methodology by clarifying or elaborating on those aspects of the method which reviewers might be unfamiliar with. It is precisely this dilemma that led to the production of Elliott et al.'s (1998) frequently cited article on publishability criteria for qualitative research. The truth is also that some journals may be more open to innovative papers, because the editors and/or reviewers in those same journals may be less focused on established methods and more interested in innovative elements (CPR is one of those journals). In general, our experience is that good research will find its way to an appropriate publishing platform and sooner or later will be recognised by the field, sometimes as prototypical, frequently-cited studies.

The Art of the Catchy Category Name. The presentation of findings is a separate issue. Findings have to not only be the result of credible and rigorous work, but they also have to be reported in a manner that sufficiently resonates with readers, in particular practising therapists and psychotherapy researchers. Findings in QPR, which often take the form of themes or categories, therefore have to be 'rich' in informing our understanding of psychotherapeutic phenomena. They should be organised clearly into narratives, using illustrative examples (for example, quotes) that are unambiguous and clear. They should also have a certain poetry and structure in themselves. For instance, if the findings are organised/presented as categories, they should use the same grammatical form for each category, such as only adjectives ("lost"/“confident"), or only verb gerund forms ("faking"/"breaking"). If the findings consist of sentences, then they should have roughly the same length. The findings (for instance, categories/ themes/ short sentences) should also use the same level of abstraction.

To come up with truthful, credible and yet catchy and resonating categories/ themes is a craft and an art. The QPR researcher thus has to become a persuasive narrator, a "strong poet" (Rorty, 1989), which sometime comes only with experience, as younger researchers may not have the confidence to distil findings until they resonate not only with themselves but also prospectively with readers within the field. The presence or absence of a good, catchy and well-resonating presentation of findings is often a make or break issue, first for reviewers, and later on for readers. The manner in which findings are presented determines whether the study is not only seen as credible (face validity), but 
whether it also illuminates phenomena in a way that broadens the reader's perspective on therapy, or has an impact on therapy practice.

\section{Qualitative Meta-Analysis: The Wave of the Future}

One other issue that we want to point to is the emergence of qualitative meta-analysis in QPR. Given that some of the first uses of quantitative meta-analysis were in the field of psychotherapy research, and that meta-analyses have subsequently become an important part of psychotherapy research, it is perhaps not that surprising that meta-analysis has also interested qualitative psychotherapy researchers (Levitt, Pomerville, \& Surace, 2016; Timulak, 2007; 2009). The emergence of qualitative meta-analysis (QMA) in QPR is attributable to the growth in number of QPR studies and thus a need to synthesise findings from these studies. Early qualitative meta-analyses of QPR studies were, however, met with suspicion and caution (this was the experiences of one of us who was involved in early efforts to publish QMAs). Nevertheless, psychotherapy as a discipline produces high numbers of qualitative studies and also has a tradition of systematically collating studies and making sense of them (for instance, in terms of policy shaping; this is perhaps surpassed only in nursing where QMA is most developed); thus, various forms of QMA have become a natural part of the landscape and will probably grow, which we see as a healthy development. QMA is also emerging as a robust way of addressing the issue of generalisability and transferability discussed earlier.

\section{Conclusion}

In this paper, we have reflected on some of the issues we encountered as researchers, authors, reviewers and editors of journals publishing QPR studies. We argue here that QPR has distinct features shaped not only by developments in qualitative methodologies, but also by the discipline of psychotherapy on its own and the unique tradition that QPR has developed over the past 30 years. QPR remains a creative endeavour that we believe can benefit from continuing reflection both on the discipline it studies and its own tradition as a specific sub-field of broader psychotherapy research. Within this tradition, QPR offers as Bill Stiles (2003, p. 494) puts it "greater scope and freedom but ...require(s) more critical thought and ...rigor" than the still more dominant hypothesestesting, quantitative approaches to psychotherapy research.

\section{References}

Barker, C., Pistrang, N., \& Elliott, R. (2015). Research methods in clinical psychology: An introduction for students and practitioners ( $3^{\text {rd }}$ ed.). Chichester, United Kingdom: Wiley.

Berzon, B., Pious, C. \& Farson, R. C. (1963). The therapeutic event in group psychotherapy: A study of subjective reports by group members. Journal of Individual Psychology, 19, 204-212.

Bloch, S. \& Reibstein, J. (1980). Perception by patients and therapist of therapeutic factors in group psychotherapy. British Journal of Psychiatry, 137, 274-278. doi: 10.1192/bjp.137.3.274

Bloch, S., Reibstein, J., Crouch, E., Holroyd, P., \& Themen, J. (1979). A method for the study of therapeutic factors in group psychotherapy. British Journal of Psychiatry, 134, 257-263. doi: 10.1192/bjp.134.3.257 
Braun, V., \& Clarke, V. (2006). Using thematic analysis in psychology. Qualitative Research in Psychology, 3, 77-101. doi: 10.1191/1478088706qp063oa

Cook, T.D., \& Campbell, D.T. (1979). Quasi-experimentation: Design and analysis issues for field settings. Chicago, IL: Rand McNally.

Elliott, R. (1989). Comprehensive process analysis: Understanding the change process in significant therapy events. In: Packer, M.J., Addison, R.B. (Eds.): Entering the circle: Hermeneutic investigation in psychology. Albany, NY: State University of New York Press.

Elliott, R., Fischer, C., \& Rennie, D. (1999). Evolving guidelines for publication of qualitative research studies in psychology and related fields. British Journal of Clinical Psychology, 38, 215-229. doi: 10.1348/014466599162782

Elliott, R., Partyka, R., Wagner, J., Alperin, R. \& Dobrenski. R., Messer, S.B., Watson, J.C. \& Castonguay, L.G. (2009). An Adjudicated Hermeneutic Single-Case Efficacy Design of Experiential Therapy for Panic/Phobia. Psychotherapy Research, 19, 543557. doi: 10.1080/10503300902905947

Elliott, R., Shapiro, D.A., Firth-Cozens, J., Stiles, W.B., Hardy, G., Llewelyn, S.P, \& Margison, F. (1994). Comprehensive process analysis of insight events in cognitive-behavioral and psychodynamic-interpersonal therapies. Journal of Counseling Psychology, 41, 449-463.

Elliott, R. \& Timulak, L. (2005). Descriptive and interpretive approaches to qualitative research. In J. Miles \& P. Gilbert (Eds.), A Handbook of research methods in clinical and health psychology (pp. 147-159). Oxford, United Kingdom: Oxford University Press.

Glaser, B. G. (1978). Theoretical sensitivity. Mill Valley, CA: Sociology Press.

Glaser, B. G., \& Strauss, A. L. (1967). The discovery of grounded theory: Strategies for qualitative research. Chicago, IL: Aldire.

Greenberg, L. S. (2007). A guide to conducting a task analysis of psychotherapeutic change. Psychotherapy Research, 17, 15-30. doi: 10.1080/10503300600720390

Hill, C. E., Thompson, B. J., \& Williams, E. N. (1997). A guide to conducting consensual qualitative research. The Counseling Psychologist, 25, 517-572. doi: 10.1177/0011000097254001

Ladany, N., Thompson, B. J., \& Hill, C. E. (2012). Cross-analysis. In C. E. Hill (Ed.), Consensual qualitative research: A practical resource for investigating social science phenomena (pp. 117-134). Washington, DC: American Psychological Association.

Lather, P. (1991). Getting smart: Feminist research and pedagogy with/in the postmodern. New York, NY: Routledge.

Levitt, H. M. (2015). Qualitative psychotherapy research: The journey so far and future directions. Psychotherapy, 52, 31-37. doi: 10.1037/a0037076

Levitt, H. M., Bamberg, M., Creswell, J. W., Frost, D. M., Josselson, R., \& SuárezOrozco, C. (2018). Journal article reporting standards for qualitative primary, qualitative meta-analytic, and mixed methods research in psychology: The APA Publications and Communications Board task force report. American Psychologist, 73, 26-46. doi: 10.1037/amp0000151

Levitt, H. M., Motulsky, S. L., Wertz, F. J., Morrow, S. L., \& Ponterotto, J. G. (2017). Recommendations for designing and reviewing qualitative research in psychology: 
Promoting methodological integrity. Qualitative psychology, 4, 2-22. doi: 10.1037/qup0000082

Levitt, H. M., Pomerville, A. \& Surace, F. I. (2016). A qualitative meta-analysis examining clients' experiences of psychotherapy: A new agenda. Psychological Bulletin, 142, 801-830. doi: 10.1037/bul0000057

Levitt, H. M., Pomerville, A., Surace, F. I., \& Grabowski, L. M. (2017). Meta-method study of qualitative psychotherapy research on clients' experiences: Review and recommendations. Journal of Counseling Psychology, 64, 626-644. doi: $10.1037 /$ cou0000222

Lipkin, S. (1954). Clients' feelings and attitudes in relation to the outcome of clientcentered therapy. Psychological Monographs: General and Applied, 68(1).

McLeod, J. (2011). Qualitative research in counselling and psychotherapy $\left(2^{\text {nd }}\right.$ ed.) London, United Kingdom: Sage.

Morrow, S. L. (2005). Quality and trustworthiness in qualitative research in counseling psychology. Journal of Counseling Psychology, 52, 250-260. doi: 10.1037/00220167.52.2.250

O’Brien, K., O'Keeffe, N., Cullen, H., Durcan, A., Timulak, L., \& McElvaney, J. (2017). Emotion-focused perspective on generalised anxiety disorder: A qualitative analysis of clients' in-session presentations. Psychotherapy Research. Ahead-of-print. doi: 10.1080/10503307.2017.1373206

Orlinsky, D. E., \& Howard, K. I. (1975). Varieties of psychotherapeutic experience: multivariate analysis of patients' and therapists reports. New York, NY: Teachers College Press.

Ponterotto, J. G. (2005). Qualitative research in counseling psychology: A primer on research paradigms and philosophy of science. Journal of Counseling Psychology, 52(2), 126-136. doi: 10.1037/0022-0167.52.2.126

Potter, J. (1996). Discourse analysis and constructionist approaches: theoretical background. In J.T.E. Richardson (ed.), Handbook of qualitative research methods for psychology and the social sciences (pp. 125-140). Leicester, United Kingdom: BPS Books.

Rennie, D. L. (2000). Grounded theory methodology as methodical hermeneutics: Reconciling realism and relativism. Theory \& Psychology, 10(4), 481-502. doi: $10.1177 / 959354300104003$

Rennie, D. L., \& Fergus, K. D. (2006). Embodied categorizing in the grounded theory method: Methodical hermeneutics in action. Theory \& Psychology, 16, 483-503. doi: $10.1177 / 0959354306066202$

Rennie, D.L., Phillips, J.R., \& Quartaro, G.K. (1988). Grounded theory: A promising approach to conceptualization in psychology? Canadian Psychology, 29, 139-150. doi: $10.1037 / \mathrm{h} 0079765$

Rogers, C.R. (1942). Counseling and psychotherapy. Cambridge, MA: Houghton Mifflin.

Rorty, R. (1989). Contingency, irony, and solidarity. Cambridge, United Kingdom: Cambridge University Press.

Smith, B. (2018). Generalizability in qualitative research: misunderstandings, opportunities and recommendations for the sport and exercise sciences. Qualitative 
Research in Sport, Exercise and Health, 10, 137-149, doi: 10.1080/2159676X.2017.1393221

Smith, J.A., Flowers, P., \& Larkin, M. (2009). Interpretative phenomenological analysis: Theory, method and research. London, United Kingdom: Sage.

Stiles, W. B. (2003). Qualitative research: Evaluating the process and the product. In S. Llewellyn \& P. Kennedy (Eds.), Handbook of clinical health psychology (pp. 477500). Chichester, United Kingdom:Wiley.

Stiles, W. B. (2011). Coming to terms. Psychotherapy Research, 21(4), 367-384. doi: $10.1080 / 10503307.2011 .582186$

Strauss, A., \& Corbin, J. (1998). Basics of qualitative research: Techniques and procedures for developing grounded theory ( 2 nd ed.). Thousand Oaks, CA: Sage.

Strupp, H. H., Wallach, M. S., \& Wogan, M. (1964). Psychotherapy experience in retrospect: Questionnaire survey of former patients and their therapists. Psychological Monographs: General and Applied, 78 (11).

ten Have, P. (1999). Doing conversation analysis: A practical guide. London, United Kingdom: Sage.

Thorne, S. (2008). Interpretive description. New York, NY: Routledge.

Timulak, L. (2007). Identifying core categories of client identified impact of helpful events in psychotherapy - a qualitative meta-analysis. Psychotherapy Research, 17, 305-314. doi: 10.1080/10503300600608116

Timulak, L. (2009). Qualitative meta-analysis: a tool for reviewing qualitative research findings in psychotherapy. Psychotherapy Research, 19, 591-600. doi: $10.1080 / 10503300802477989$

Timulak, L. (2012). Review of the book 'Consensual Qualitative Research', edited by Clara Hill. Psychotherapy, 49, 273-274. doi: 10.1037/a0028025

Wertz, F.J. (1983). From everyday to psychological description: Analyzing the moments of a qualitative data analysis. Journal of Phenomenological Psychology, 14, 197241. 